\title{
Desenvolvimento motor e autoconceito de escolares com transtorno do desenvolvimento da coordenação
}

\author{
Thais Silva Beltrame \\ Universidade do Estado de Santa Catarina - Florianópolis - Santa Catarina - Brasil \\ Fernando Luiz Cardoso \\ Universidade do Estado de Santa Catarina - Florianópolis - Santa Catarina - Brasil \\ Juliano Maestri Alexandre \\ Universidade do Estado de Santa Catarina - Florianópolis - Santa Catarina - Brasil \\ Carla Simon Bernardi \\ Universidade do Estado de Santa Catarina - Florianópolis - Santa Catarina - Brasil
}

\begin{abstract}
Resumo
Verificou-se a influência de um programa de intervenção motora no desenvolvimento motor e autoconceito de escolares com Transtorno do Desenvolvimento da Coordenação (TDC). Participaram do programa de intervenção de 16 semanas, 12 crianças com idades de 10 anos $( \pm 6$ meses), divididas em dois grupos;sete escolares com indicativo TDC (GT) e cinco escolares com risco para TDC (GR). Utilizou-se a bateria motora MABC-2 (Henderson, Sugden, \& Barnett, 2007) e a Escala de Auto-percepção para Crianças (Harter, 1982). Para analisar os efeitos da intervenção, utilizou-se o teste de Wilcoxon, o nível de significância foi de $p>0,05$. O programa se mostrou efetivo quando observado o TDC proporcionando influência positiva e significante nos valores das pontuações totais, pontuação padrão e percentis do Teste MABC-2. Entretanto, para o autoconceito essa efetividade não foi totalmente confirmada.
\end{abstract}

Palavras-chave: Desenvolvimento infantil; criança; autoconceito.

\section{Motor development and self-concept of children with Developmental Coordination Disorder}

\begin{abstract}
It was verified the influence of a motor intervention program in motor development and school of self with Developmental Coordination Disorder (DCD). Participated in the 16-week intervention program, 12 children aged 10 years $( \pm 6$ months), divided into two groups; seven children with indicative TDC (GT) and five children with risk for TDC (GR). We used the motor battery MABC-2 (Henderson, Sugden, \& Barnett, 2007) and Selfawareness Children Scale (Harter, 1982). To analyze the effects of the intervention, we used the Wilcoxon test, the level of significance was set at $p>0.05$. The program was effective when observing the TDC providing a positive and significant influence on the total score values, standard scores and percentiles MABC-2 test. However, for this self-effectiveness has not been fully confirmed.
\end{abstract}

Keywords: Child development; child; self concept.

\section{Desarrollo motor y auto concepto de escolares con trastorno del desarrollo}

\section{de la coordinación}

\section{Resumen}

Se verificó la influencia de un programa de intervención motora en el desarrollo motor y auto concepto de escolares con Trastorno del Desarrollo de la Coordinación (TDC). Participaron del programa de intervención de 16 semanas 12 niños con edades de 10 años ( \pm 6 meses), divididas en dos grupos; siete escolares con indicativo TDC (GT) y cinco escolares con riesgo para TDC (GR). Se utilizó la batería motora MABC-2 (Henderson, Sugden, \&Barnett, 2007) y la Escala de Auto-percepción para Infantes (Harter, 1982). Para analizar los efectos de la intervención, se utilizó el test de Wilcoxon, el nivel de significancia fue de $p>0,05$. El programa se mostró efectivo cuando observado el TDC proporcionando influencia positiva y significante en los valores de las puntuaciones totales, puntuación patrón y percentis del Test MABC-2. Sin embargo, para el autoconcepto esa efectividad no fue totalmente confirmada.

Palabras clave: Desarrollo infantil; niño; auto concepto. 


\section{Introdução}

As dificuldades e comprometimentos nos movimentos ou atraso no alcance dos marcos motores, na maturação e aquisição das habilidades básicas locomotoras, fundamentais e estabilizadoras, pode produzir efeito significativamente negativo no desenvolvimento global, assim como em atividades de vida diária, relações sociais, emotivas, afetivas e no ambiente escolar (Wright \& Sugden, 1996). A criança que apresenta dificuldades no desempenho de habilidades motoras fundamentais diminui as chances de participação em atividades motoras escolares, reduzindo seu engajamento motor e a participação futura em atividades que asseguram a qualidade de vida (Valentini, 2002a).

Uma das dificuldades do movimento refere-se ao transtorno da coordenação motora que se caracteriza por um prejuízo na coordenação e no controle de movimentos básicos e por um desempenho abaixo do nível esperado em atividades que exigem coordenação motora (Oliveira, Loss, \& Petersen, 2005). Na década de 1980, esses problemas de dificuldade de movimento e coordenação foram reconhecidos pela Organização Mundial da Saúde - OMS e pela Associação Americana de Psiquiatria - APA, e receberam o nome de Transtorno do Desenvolvimento da Coordenação TDC (APA, 1995).

O TDC não é simplesmente um problema de coordenação específico da infância, que desaparece gradualmente, de forma natural, com o crescimento e a maturação, pois há evidências de que as dificuldades motoras podem, ao longo da vida, levar ao isolamento, à depressão e à marginalização (Hellgreen \& cols., 1994; Gillberg, 2000; Dewey, Kaplan, Crawford, \& Wilson, 2002; Cantell, Smyth, \& Ahonen, 1994; Polatajko, 2003). É um distúrbio pode ser observado no atraso motor e na dificuldade com que a criança realiza movimentos básicos, como pegar objetos, engatinhar e sentar, posteriormente, esse comportamento descoordenado evidencia-se em tarefas mais complexas (Valentini \& cols., 2012).

Autores afirmam que o TDC representa um dos maiores problemas de saúde para a criança, pois sua prevalência na população mundial provavelmente é maior do que $6 \% \mathrm{em}$ crianças de 5 a 11 anos (Dewey \& Wilson, 2001), uma vez que somente uma pequena parte das crianças é identificada (Hay, Hawes, \& Faught, 2004). A criança incapaz de participar das atividades típicas dos colegas da mesma idade pode sofrer problemas sociais e emocionais, porque a idade escolar é uma fase da vida na qual ela conquista seu lugar no grupo, por meio da participação em brincadeiras relacionadas a atividades físicas (Dewey \& cols., 2002; Segal, Mandich, Polatajko, \& Cook., 2002; Cantell \& cols.,1994).

Pode-se afirmar que crianças com dificuldades motoras são incapazes de participar de muitas atividades físicas cujos colegas participam, mas o mais importante é que podem ter dificuldades em atividades em casa, na escola e na vida social e, por consequência, reduzir sua percepção de competência e motivação para participar das oportunidades que Ihes são apresentadas (Hands, 2007). Esta percepção aponta os sentimentos que a criança possui em relação a si mesma e aos rótulos que são colocados em comparação com a sociedade (Harter, 1984). O autoconceito é um termo mais abrangente relativo à descrição de um indivíduo, definindo-se como o conhecimento que o indivíduo tem de si próprio, englobando aspectos cognitivos, afetivos e comportamentais (Sisto, Bartholomeu, Rueda, \& Fernandes, 2004), acerca das suas capacidades, competências, aparência física e aceitabilidade social (Byrne, 1984). Também é considerado por muitos autores como uma das principais qualidades protetoras individuais preditivas de um bom funcionamento psicossocial (Novaes, 2003; Campos, 2004).

Com essa relação entre o TDC e o autoconceito, uma intervenção é recomendada visto que é indicada para indivíduos com deficiências ou déficit motor; sua finalidade, seguindo uma abordagem desenvolvimentista, deve atender às principais necessidades do aluno, promovendo a interação dinâmica entre as características do executante, da tarefa e do ambiente, objetivando o aumento do repertório motor (Gallahue \& Ozmun, 2005).

Diante deste contexto referente ao comportamento motor, firma-se a seguinte questão problema: "Qual a influência de um programa de intervenção motora no desenvolvimento motor e autoconceito de escolares com indicativo e risco de Transtorno do Desenvolvimento da Coordenação Motora?

\section{Método}

Este estudo caracterizou-se como quase-experimental (Thomas \& Nelson, 2002) e o delineamento da pesquisa constituiu-se de três fases: pré-teste, execução do programa de intervenção motora e pós-teste. Os participantes foram selecionados por conveniência em uma comunidade escolar da mesorregião da Grande Florianópolis-SC a 6 km da capital. A instituição de ensino atende estudantes com idades entre 6 e 18 anos, nas séries do ensino fundamental, no período matutino e vespertino e fica situada em uma área privilegiada e acessível do município, integrando um corpo discente de todas as regiões da cidade e dos municípios vizinhos (Florianópolis, São José, Palhoça e Biguaçu).

Os participantes deste estudo foram previamente identificados no estudo de Silva (2009), pesquisadora do Laboratório de Distúrbios da Aprendizagem e do Desenvolvimento - LADADE, da Universidade do Estado de Santa Catarina - UDESC que investigou o desempenho motor, desempenho acadêmico e senso de auto-eficácia de escolares. Considerando o expressivo apoio e interesse da instituição em dar continuidade à pesquisa, o presente estudo propôs-se a dar sequenciamento e verificar as alterações no desempenho motor destes escolares após um programa de intervenção motora. Para a inclusão das crianças no estudo, foram estabelecidos os critérios: possuir percentil igual ou inferior a 15, segundo protocolo do Teste MABC-2; possuir Termo de Consentimento Livre e Esclarecido assinado pelo responsável; estar regularmente matriculado e ser favorável a participar de 21 sessões de intervenção motora. Como 
critérios de exclusão consideraram-se: não demonstrar interesse em participar do estudo; desistência ou retirada do consentimento dos pais ou responsáveis; não comparecer (faltar) às sessões de intervenção motora; não apresentar integridade física e mental.

Algumas limitações com relação à amostra ocorreram: uma epidemia de gripe $\mathrm{H} 1 \mathrm{~N} 1$ no estado no período da intervenção, o que acarretou um atraso na pesquisa e grande perda amostral. Outros sete escolares retiraram-se do experimento por motivos particulares. Assim, a composição final constituiu-se por 12 escolares de $3^{\mathrm{a}}$ a $5^{\mathrm{a}}$ séries que obtiveram risco ou indicativo de TDC conforme avaliação no MABC-2.

Destes 12 escolares, cinco pertenciam ao Grupo com Risco para TDC (GR) e sete ao Grupo com Indicativo de TDC (GT). O grupo GR foi composto por três escolares do sexo feminino e dois do sexo masculino. O grupo GT foi composto por três escolares do sexo feminino e quatro do sexo masculino. A média de idade geral e dos grupos foi de 10 anos, sendo \pm 6 meses para o geral e \pm 8 meses para GR; não houve desvio padrão para o GT.

\section{Instrumentos}

Para a coleta dos dados utilizoua Bateria para Avaliação do Movimento de Crianças o Movement Assessment Battery for Children - MABC-2 (Henderson, Sugden, \& Barnett, 2007), a Escala de Autopercepção para Crianças (Self-Perception Profile for Children-SPPC) (Harter, 1982) e a Ficha de Registro e Identificação Escolar.

O MABC-2 consiste em uma lista de checagem e um teste de desempenho motor aplicável em clínicas e escolas para detectar dificuldades motoras em crianças de 4 a 16 anos. O teste possui quatro conjuntos de tarefas, cada um apropriado a uma faixa etária específica, sendo a Faixa 1 para testes com crianças de 4 a 6 anos, Faixa 2 para crianças de 7 a 10 anos, e Faixa 3 para crianças de 11 a 16 anos.
Os testes são divididos nas categorias: Testes de Destreza Manual, Testes de Habilidades com Bola e Testes de Equilíbrio. As tarefas são diferenciadas para as idades, levando em conta o padrão de desenvolvimento da criança em cada faixa etária conforme ilustra o Quadro 1.

Para a realização dos testes foram utilizados Formulários de Registro nos quais foram armazenados os dados pessoais dos alunos como nome, idade, endereço residencial entre outros, e o desempenho nas tarefas propostas pelo teste. Os formulários de registro possuem também um espaço para a realização de uma avaliação qualitativa, que leva em conta as influências comportamentais da criança durante a realização dos testes. Após a aplicação dos testes, os resultados foram somados, primeiramente dentro de cada conjunto de tarefas, para ser possível obter o escore das Destrezas Manuais Totais, Habilidades com Bola Totais e Equilíbrio Total. Em seguida foram somados esses três últimos valores, para se obter o MABC-2 Total de cada criança.

Os valores do MABC-2 Total foram comparados à tabela de percentis, presentes no protocolo do teste. As crianças cujo MABC-2 totais apresentaram um resultado a baixo do $5^{\circ}$ percentil foram consideradas com indicativo de Transtorno do Desenvolvimento da Coordenação - TDC. Os valores entre o $5^{\circ}$ e o $15^{\circ}$ percentil indicaram que a criança tem risco de apresentar dificuldades motoras. Valores acima do $15^{\circ}$ percentil indicaram que não foi detectada nenhuma dificuldade motora. O teste é administrado individualmente, e leva cerca de 20 a 40 minutos para ser realizado dependendo da idade e da dificuldade motora apresentada pela criança. O presente estudo utilizou as faixas 2 e 3 .

A confiança de teste reteste do MABC foi considerada boa (Henderson \& Sugden, 1992). Croce, Horvat e McCarthy (2001) observaram a confiança do teste reteste do $M A B C$, verificando um coeficiente de correlação intra-classe alto em todos os grupos de idades. Ao comparar o MABC com o teste Bruininks-Oseretsky Test of Motor Proficiency (BOMPT), nas versões (longa e curta), foi demonstrada uma

Quadro 1. Testes segundo as categorias de habilidades motoras (MABC-2).

\begin{tabular}{|c|c|c|c|c|}
\hline \multicolumn{2}{|l|}{ Categorias } & \multirow{2}{*}{$\begin{array}{c}\text { Testes Faixa } 2 \\
\text { Colocando pinos }\end{array}$} & \multirow{2}{*}{$\frac{\text { Testes Faixa } 3}{\text { Invertendo pinos }}$} & \multirow{2}{*}{$\frac{\text { Registro }}{\text { Tempo }}$} \\
\hline & DM1 & & & \\
\hline \multirow[t]{2}{*}{ Destreza Manual } & DM2 & Passando cordão & Triângulo com porcas & Tempo \\
\hline & DM3 & Trilha da bicicleta & Trilha da bicicleta & $\mathrm{N}^{\circ}$ de acertos \\
\hline \multirow{2}{*}{$\begin{array}{l}\text { Lanças e } \\
\text { Receber }\end{array}$} & LR1 & $\begin{array}{l}\text { Lançar e receber com as } \\
\text { duas mãos }\end{array}$ & Lançar e receber com uma mão & $\mathrm{N}^{\circ}$ de acertos \\
\hline & LR2 & Lançar saco de feijão & Lançar no alvo & $\mathrm{N}^{\circ}$ de acertos \\
\hline \multirow{3}{*}{ Equilíbrio } & EQ1 & Equilíbrio sobre a tábua & Equilíbrio sobre duas tábuas & Tempo \\
\hline & EQ2 & Andando sobre a linha & Caminhando para trás & $N^{\circ}$ de erros \\
\hline & EQ3 & Saltando tapetes & Saltando tapetes em zig-zag & $\mathrm{N}^{\circ}$ de erros \\
\hline
\end{tabular}


validade concorrente moderada (Crawford \& cols., 2001; Croce \& cols., 2001). Entretanto, o MABC objetiva verificar limitações motoras, enquanto que o objetivo do BOMPT é caracterizar a performance motora, sendo assim, uma completa concordância não é necessariamente esperada (Henderson \& Sugden, 1992). Estes resultados suportam o uso do MABC como medida de habilidade motora em crianças com idade de 5 a 12 anos (Croce \& cols., 2001). O MABC como instrumento ainda obteve a melhor avaliação ao ser considerado em uma revisão de critérios diagnósticos para distúrbios de desenvolvimento motor (Geuze, Jongmans, Scoemaker, \& Smits-Engelsman, 2001). Portanto, justifica-se a utilização do MABC-2 no objetivo proposto.

A Escala de Autopercepção para Crianças (Self-Perception Profile for Children - SPPC) é a nova versão da "Perceived Competence Scale for Children" (Harter, 1982) e é formada por 36 itens divididos em seis sub-escalas, onde cada item é composto por duas afirmações, interligadas com o conectivo "mas". Em cada sub-escala três itens estão organizados de forma que a primeira afirmação represente alta competência ou adequação (sinalizadas abaixo com o sinal +) e os outros três de forma que a primeira afirmação represente baixa competência ou adequação (sinalizados abaixo com o sinal). As seis sub-escalas são: a) Competência escolar/acadêmica (itens 1+, 7+, 13-, 19-, 25+ e 31-): avalia a percepção da criança em relação à sua competência diante das demandas escolares, por exemplo, como é seu desempenho na sala de aula e quão inteligente se julga ser; b) Aceitação Social (itens 2-, 8+, 14-, 20+, 26-, 32+): investiga o grau com que a criança é aceita por seus pares, o quanto se sente popular, quantidade de amigos e facilidade para se relacionar; c) Competência Atlética (itens 3+, 9-, 15+, 21+, 27-, 33-): avalia a percepção da criança acerca de suas habilidades para os esportes, por exemplo, sentimento de quão bom é seu desempenho em esportes e atividades atléticas; d) Aparência Física (itens 4+, 10+, 16-, 22-, 28-, 34+): verifica o grau de satisfação da criança com sua aparência, se gosta do seu corpo e se o considera bonito; e) Comportamento (itens 5-, 11+, 17+, 23-, 29-, 35+): avalia o nível em que a criança está satisfeita com o seu comportamento, se age de forma correta e evita situações-problema e f) Mais-valia Global (itens 6-, 12-, 18+, 24+, 30+, 36-): avalia a extensão em que a criança está satisfeita consigo mesma, com a forma como conduz sua vida e se é, em geral, feliz. Constitui um julgamento global ao invés de uma avaliação por domínios.

Os seis primeiros itens da escala foram organizados de acordo com a ordem acima; os demais também seguem essa alternância ao longo da escala. O questionário apresentado à criança possui o título "Como eu sou". Cada item é composto por duas afirmações e a criança deve escolher qual delas melhor a descreve. Em seguida, deve pensar se a afirmação escolhida é pouco verdadeira ou muito verdadeira para ela. A pontuação é feita no formato Likert, atribuindo-se os valores 4, 3, 2 ou 1; representando o escore 4 o maior senso de adequação. Os escores são somados para que se tenha uma pontuação final de cada sub-escala e a partir daí se tem o perfil de cada criança. O questionário pode ser admi- nistrado em grupo ou individual de acordo com Novaes (2003) e será adotado o critério de classificação de Teixeira (2008).

Os dados de fidedignidade relatados por Harter (1985) determinam que na amostra com crianças de $6^{a}$ e $7^{a}$ séries a fidedignidade foi de 0.84 , com crianças de $3^{a}$ a $6^{a}$ séries foi de 0.81 e consistência interna variando entre 0.55 e 0.93 . Estudos de validação da escala adaptada para o Brasil apresentaram índices semelhantes aos dados americanos para cada sub-escala (Bandeira, Reppold, Pflüger, \& Facio, 2003). Em outro estudo também com crianças brasileiras (Villwock, 2005), a fidedignidade encontrada foi de 0.83, compatível coma apresentada pela autora do instrumento (Harter, 1985). A escala SPPC (Harter, 1985) foi submetida à validação de conteúdo (Vallerand, 1989), em crianças brasileiras com idade entre 8 e 10 anos, através de cinco etapas: versão preliminar, aplicação na população, avaliação de clareza e da pertinência, da fidedignidade e da validade de construção. Os resultados indicaram que a versão preliminar atendeu aos critérios de clareza e pertinência esperada e que correlações de média a forte para validade e fidedignidade foram encontradas, semelhantes aos resultados apresentadas pela autora do instrumento (Villwock, 2005).

A ficha de registro e identificação escolar é um documento oficial de uso da instituição escolar e obteve-se permissão para pesquisar neste arquivo informações como: renda familiar, profissão dos pais, histórico de repetência escolar, endereço, data de nascimento, escolaridade dos pais, situação conjugal e com quem a criança mora.

\section{Procedimentos para a Coleta}

A coleta de dados teve início após a aprovação da instituição escolar e do Comitê de Ética em Pesquisa em Seres Humanos - CEP/UDESC 1 . As avaliações iniciaram-se após a reunião com os responsáveis legais das crianças que obtiveram indicativo de dificuldades motoras, onde apresentaram-se os objetivos, procedimentos e demais esclarecimentos. Não fizeram parte do estudo crianças cujos responsáveis ou professores relataram problemas físicos ou mentais. Após as avaliações, as crianças que mantiveram percentis igual ou inferior a 15 no MABC-2 total participaram das intervenções. Ao término da $21^{\text {a }}$ sessão os escolares foram reavaliados tanto no desempenho motor como no autoconceito. Os resultados individuais foram repassados para os pais através de relatório individual.

\section{O Programa de Intervenção}

O período de Coleta e Intervenção, para ambos os grupos, foi de 16 semanas; cada participante recebeu 21 sessões, individuais, totalizando 252 sessões de intervenção motora. As sessões aconteceram nos períodos das aulas de educação física, realizadas duas vezes por semana, durante

1 Sob o node registro 23/2009/ resolução 196/96 e 251/97 do Conselho Nacional de Saúde. 
45 minutos, no espaço escolar, ministradas pela pesquisadora, uma bolsista PIBIC e uma profissional de educação física e foram idênticas no sequenciamento e conteúdo, tornando-se assim uma variável de controle.

As intervenções foram organizadas de acordo com os três grupos de habilidades sugeridas no MABC2 (destreza manual, lançar e receber, e equilíbrio). Para cada sessão houve um plano de aula e um anedotário, onde registraram-se observações da criança.O programa de intervenção foi estruturado dentro de uma perspectiva de Desenvolvimentista e nos pilares do estilo de ensino Descoberta Orientada que busca promover um relacionamento particular entre professor-aluno, no qual a sequência de tarefas propostas ocasiona uma sequência correspondente de resposta pelo aprendiz. Cada tarefa ou desafio deve provocar uma única resposta correta, descoberta pelo aluno. Caso isto não aconteça, o professor acrescenta outras tarefas ou dicas que conduzam o aluno a resposta certa. O efeito cumulativo dessa sequência, ou seja, um processo convergente, leva o aluno a descobrir o conceito, princípio ou idéia procurado (Krug, 2009). As avaliações e intervenções foram realizadas na quadra poliesportiva e em uma sala com $30 \mathrm{~m}^{2}$, ventiladores de tetos e próxima ao bebedouro e sanitários.

\section{Análise dos Dados e Tratamento Estatísticos}

Para descrever o desenvolvimento motor e o autoconceito, utilizou-se estatística descritiva, incluindo medidas de tendência central (média, mediana e moda) e variabilidade (desvio-padrão, variação e freqüências), bem como os escores fornecidos pelos testes.Considerando que o $\mathrm{n}$ amostral ficou reduzido a doze participantes, optou-se por utilizar estatística não paramétrica para as análises inferenciais, reduzindo a chance de cometer erros do tipo II.Para verificar as alterações provocadas pelo programa de intervenção motora nas variáveis dependentes do estudo (desempenho nos testes motores e autoconceito), utilizou-se o teste não paramétrico de Wilcoxon. O nível de significância adotado foi $p<0,005$. Os dados foram tabulados no programa SPSS for Windows e realizou-se a análise dos resultados através de três critérios distintos: escore padrão, percentil e avaliação descritiva. Para este estudo optou-se por analisar os dados utilizando como critério a pontuação padrão, pois já contabiliza as idades; e os demais critérios se utilizaram como medidas complementares. Foram realizados relatórios finais individuais que foram repassados a escola e explicados aos pais em reunião.

\section{Resultados}

O grupo de crianças com TDC (GT) foi composto por sete participantes, com idades médias entre 10 anos $( \pm 6$ meses), quatro do sexo feminino e três do sexo masculino. O grupo denominado risco para TDC (GR) foi composto por cinco participantes, com idade média de 10 anos $( \pm 4$ meses), três do sexo feminino e dois do sexo masculino.

\section{Desenvolvimento Motor a Dificuldades Motoras}

O desenvolvimento motor obtido por meio da pontuação total do teste para o grupo GT $(47.28, \pm 5.76)$ foi considerado com "dificuldade motora significante". Já o grupo GR com média $61.80( \pm 2.77)$ foi considerado como "risco para apresentar dificuldades motoras devendo haver um monitoramento" dessas crianças. Diante destes resultados, foi possível observar menor média na pontuação padrão das habilidades de Lançar e Receber, com média de 15.6 ( \pm 3.09$)$, (Tabela 2$)$, contudo, considerando os testes separadamente a menor média foi encontrada no teste de Equilíbrio 2, com média 4.58 ( \pm 3.94$)$ conforme Tabela 3.

Constatou-se que os escolares com TDC obtiveram médias inferiores nas tarefas de equilíbrio ( $E Q 2, X=3.57, \pm$ 3.41; EQ3, $X=3.43, \pm 1.51$ ) enquanto os escolares do grupo de risco apresentaram mais dificuldades nas tarefas de destreza manual (DM1, X=5.60, \pm 2.07 ; DM2 X=5.60, \pm 0.89 ). A menor dificuldade motora foi observada na tarefa DM3, que consiste em tracejar um desenho de uma trilha de bicicleta, onde GT apresentou média 9.71( \pm 2.69$)$ e GR média 11.40 $( \pm 0.89)$. Sobre a habilidade de destreza manual, nota-se que na tarefa 2 (DM2)as crianças com TDC obtiveram média discretamente superior $(5.85, \pm 2.73)$ quando comparadas às crianças do grupo de risco $(5.60, \pm 0.89)$. Os participantes apresentaram maior dificuldade nas habilidades de Lançar e Receber. Contudo, na observação por grupo, os participantes com TDC obtiveram maior severidade nas habilidades de Equilíbrio e os participantes do grupo de risco nas habilidades de Destreza Manuais.

\section{Autoconceito}

Constatou-se que os participantes possuíam um senso de adequação "moderado" de seu autoconceito (Autoconceito total, $109.50 \pm 16.30$, e $x=3.04$ ).Ao verificar os valores descritivos das sub-escalas apresentados na Tabela 5, é possível observar um senso de adequação "moderado" nas percepções de Competência Escolar (16.67 \pm 4.64 , e x=2.7), Competência Atlética $(16.83 \pm 3.81, x=2.80)$, Comportamento (17.58 \pm 4.81 , e x 2.93). Já as pontuações das sub-escalas: Aceitação Social (18.50 \pm 4.64 , e x=3.10), Aparência Física (19.58 \pm 3.20 , e $x=3.26)$ e Mais-Valia Global (20.33 \pm 3.70 , e $x=3.39$ ) indicaram que os participantes possuem senso de adequação "mais elevado".

\section{Influência do Programa de intervenção no Desempenho Motor}

A tabela 1 apresenta os valores descritivos para o desenvolvimento motor pré e pós-programa interventivo dos participantes do estudo.

Ao observar os resultados descritos na tabela 1 é possível afirmar que para ambos os grupos (GT e GR) o programa interventivo proporcionou influência positiva e 
Tabela 1. Pontuações Gerais da Bateria MABC-2, pré e pós-programa de intervenção motora por grupo de TDC (GT) e grupo de risco para TDC (GR)

\begin{tabular}{|c|c|c|c|c|c|c|c|c|c|}
\hline & & \multicolumn{3}{|c|}{ PRÉ } & \multicolumn{3}{|c|}{ Pós } & \multicolumn{2}{|c|}{ Wilcoxon } \\
\hline & & $x$ & $d p$ & $m_{d}$ & $x$ & $d p$ & $m_{d}$ & z & $\mathbf{P}$ \\
\hline \multirow{2}{*}{$\begin{array}{c}\text { Pontuação Total } \\
\text { Teste }\end{array}$} & GT & 47.28 & 5.76 & 48.00 & 63.57 & 10.92 & 58.00 & -2.371 & 0.018 \\
\hline & GR & 61.80 & 2.77 & 61.00 & 70.20 & 6.38 & 72.00 & -2.032 & 0.042 \\
\hline \multirow{2}{*}{ Pontuação Padrão } & GT & 4.14 & 0.90 & 4.00 & 7.00 & 2.24 & 6.00 & -2.388 & 0.017 \\
\hline & GR & 6.40 & 0.55 & 6.00 & 8.00 & 1.00 & 8.00 & -2.070 & 0.038 \\
\hline \multirow{2}{*}{ Percentil } & GT & 3.00 & 1.91 & 2.00 & 19.43 & 24.64 & 9.00 & -2.375 & 0.018 \\
\hline & GR & 11.80 & 3.83 & 9.00 & 26.20 & 10.52 & 25.00 & -2.041 & 0.041 \\
\hline
\end{tabular}

GT - grupo de crianças com TDC; GR - grupo do risco para TDC.

Tabela 2. Pontuação Padrão e Intervalo de Confiança das Habilidades Motoras da Bateria MABC-2pré e pós-programa de intervenção motora.

\begin{tabular}{|c|c|c|c|c|c|c|c|c|c|c|}
\hline & \multicolumn{4}{|c|}{ PRÉ } & \multicolumn{4}{|c|}{ Pós } & \multicolumn{2}{|c|}{ Wilcoxon } \\
\hline & $x$ & Dp & $m_{d}$ & I.C.(95\%) & $x$ & $\mathrm{Dp}$ & $m_{d}$ & I.C. $(95 \%)$ & z & $\mathbf{P}$ \\
\hline DM & 20.8 & 3.10 & 20.00 & $18.9-22.8$ & 21.3 & 4.27 & 21.00 & $18.6-20.0$ & -0.357 & 0.721 \\
\hline LR & 15.6 & 3.09 & 15.00 & $13.6-17.5$ & 18.9 & 3.89 & 19.0 & $16.4-21.4$ & -2.494 & 0.013 \\
\hline$E Q$ & 17.67 & 5.71 & 17.50 & $14.0-21.3$ & 25.9 & 7.72 & 25.5 & $21.0-30.8$ & -2.675 & 0.007 \\
\hline
\end{tabular}

DM - Destreza Manual; LR - Lançar e Receber; EQ - Equilíbrio.

Tabela 3. Pontuação Padrão dos Sub-testes das Habilidades Motoras da Bateria MABC-2, pré e pós-programa de intervenção motora.

\begin{tabular}{lccccccccc}
\hline & & PRÉ & & & PÓS & & \multicolumn{2}{c}{ Wilcoxon } \\
\cline { 2 - 10 } & $\mathbf{X}$ & $\mathbf{d p}$ & $\mathbf{m}_{\mathrm{d}}$ & $\mathbf{X}$ & $\mathbf{D p}$ & $\mathbf{m}_{\mathrm{d}}$ & $\mathbf{z}$ & $\mathbf{P}$ \\
\hline DM1 & 4.92 & 1.68 & 4.50 & 5.16 & 2.82 & 6.00 & -0.254 & 0.799 \\
\hline DM2 & 5.75 & 2.09 & 6.00 & 5.42 & 3.65 & 6.00 & -0.312 & 0.755 \\
DM3 & 10.42 & 2.23 & 11.00 & 10.92 & 2.54 & 11.00 & -0.674 & 0.500 \\
LR1 & 9.75 & 3.16 & 8.00 & 10.75 & 2.30 & 11.00 & -1.027 & 0.304 \\
LR2 & 5.83 & 1.70 & 5.00 & 8.16 & 2.85 & 8.50 & -2.251 & $\mathbf{0 . 0 2 4}$ \\
\hline EQ1 & 8.17 & 3.86 & 8.50 & 9.58 & 2.47 & 8.50 & -0.970 & 0.332 \\
EQ2 & 4.58 & 3.94 & 3.00 & 7.33 & 3.77 & 8.50 & -1.798 & 0.072 \\
EQ3 & 4.92 & 2.84 & 4.00 & 9.00 & 2.95 & 9.50 & -2.810 & $\mathbf{0 . 0 0 5}$ \\
\hline
\end{tabular}


significante nos valores das pontuações totais, pontuação padrão e percentis do Teste MABC-2 segundo análise por meio do teste Wilcoxon. A análise dos dados do programa interventivo sobre as categorias de habilidades propostas pelo teste MABC-2, constatou influência significativamente positiva nas habilidades de Lançar e Receber $(p=0.013)$ e mais fortemente na habilidade de Equilíbrio ( $p=0.007$ ). Não se constatou influência positiva do programa interventivo sobre as habilidades de Destreza Manual $(p=0.721)$, conforme é possível observar na Tabela 2.

Constata-se que não houve sobreposição nos intervalos de confianças das habilidades de Lançar e Receber, e Equilíbrio. Outro achado importante é relativo ao que valores descritos na Tabela 3. Ao observarmos os testes separadamente, referentes a cada categoria de habilidade motora, só encontramos diferença significativa na aplicação do teste estatístico de Wilcoxon, no sub-teste de Lançar e Receber 2 $(p=0.024)$ e no sub-teste de Equilíbrio3 $(p=0.005)$. Contudo, é possível constatar que com exceção do sub-teste de Destreza Manual 2, todos os demais sub-testes apresentaram médias superiores no pós-teste, levando em sua somatória a uma diferença no resultado final, onde pode ser verificado nas tabelas 1 e 2 . Neste sentido, também é possível observar na tabela 4 os sub-testes segundo os grupos interventivos; onde somente o grupo com TDC apresenta diferença estatisticamente significativa no sub-teste de Equilíbrio 2.

Considera-se relevante ressaltar que ao fim do programa interventivo as crianças com TDC (GT) apresentaram aumento superior nas médias dos testes de habilidades motoras conforme ilustra a tabela 4. Outra consideração em relação aos achados deste estudo foi em relação à influência positiva nas habilidades de equilíbrio das crianças do grupo com TDC, visto que ao início o estudo esta era a habilidade da qual foram obtida menores médias, e após o programa interventivo foram nestas mesmas habilidades que observou a maior diferença significativa.

\section{No Autoconceito}

A tabela 5 apresenta os resultados da pontuação da avaliação do autoconceito total e o resultado parcial de sua categoria segundo a Escala de Autopercepção- SPPC.

Tabela 4 - Pontuação Padrão dos sub-testes das Habilidades Motoras da Bateria M-ABC2 pré e pós-programa de intervenção motora no grupo de TDC (GT) e no grupo de risco para TDC(GR).

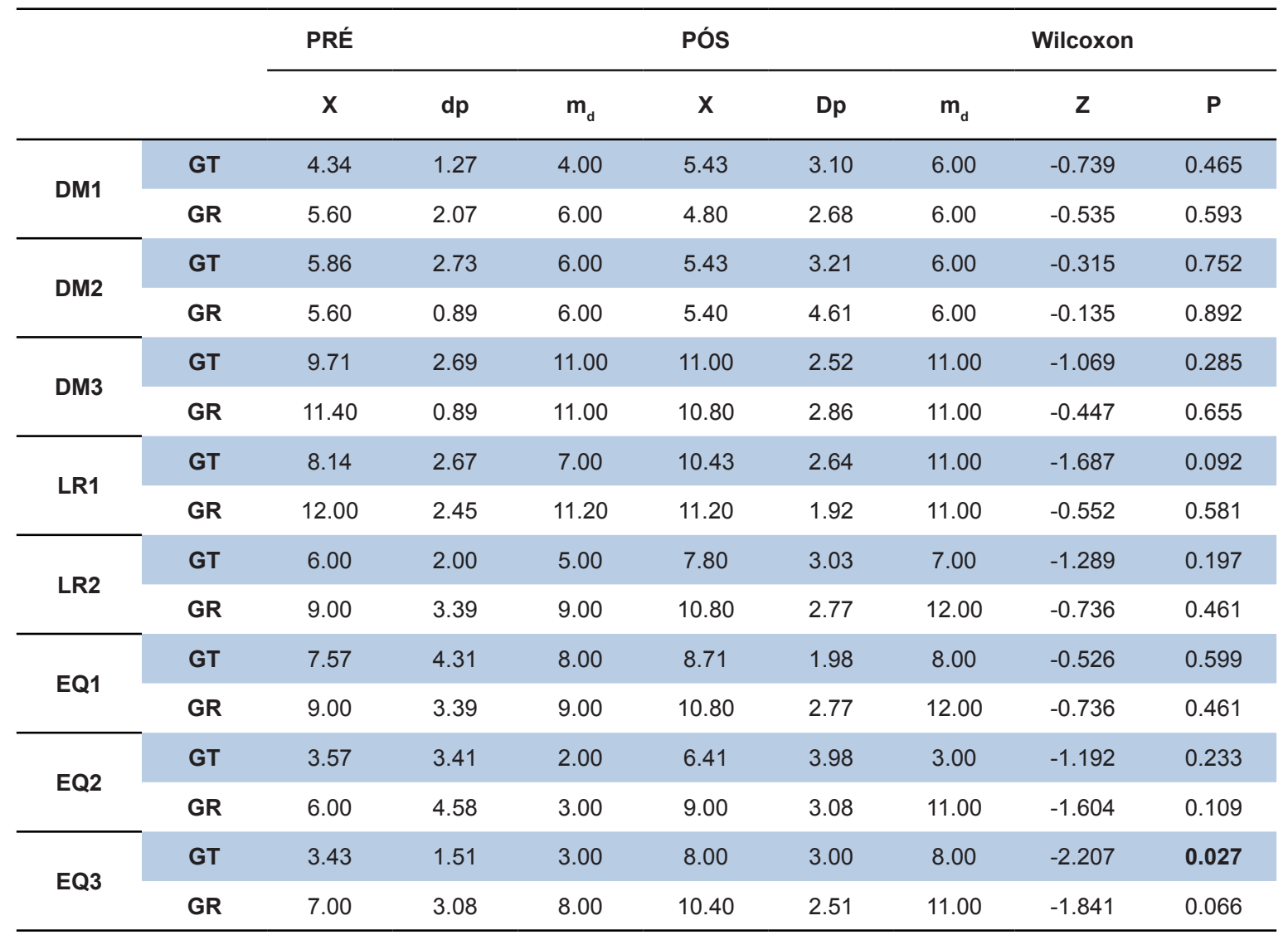


No que se refere à pontuação total desta escala, ou Autoconceito total, as análises dos resultados evidenciam uma diminuição significativa no pré-teste para pós-teste pela análise através do teste de Wilcoxon $(p=0.007)$. Contudo, essa diminuição não alterou substancialmente a média, permanecendo como "Moderado" o senso de autoconceito total dos participantes. O programa interventivo demonstrou ter influenciado negativamente as médias das categorias Aceitação Social (17.92 \pm 3.37, e $x=2.99)$, Aparência Física (16.42 \pm 5.82, e $x=2.74)$ e Mais-Valia Global (17.17 \pm 4.71 , e $x=2.86)$. No pré-teste os participantes apresentaram senso de adequação "Mais Elevada" para essas categorias e no pós-teste passou a ser "Moderada". A análise estatística por meio do teste Wilcoxon não constatou diferença estatisticamente significativa, e a Competência Atlética foi a única categoria que demonstrou aumento positivo em sua média, mesmo que sutil, após o programa interventivo; apresentando média pré igual a $16.83( \pm 4.56)$ e pós igual $16.92( \pm 2.74)$. Este dado se apresenta com maior destaque na tabela 6 , onde mostra a pontuação dos grupos interventivos. Outro achado diz respeito ao grupo de escolares com TDC (GT), indicando uma diminuição significativa na forma como julgam seu comportamento $(p=0.027)$ conforme também apresenta a tabela 6 .

Por fim, constata-se que o grupo de crianças com TDC apresentou aumento da média na sua percepção de competência atlética após a intervenção, enquanto o grupo de risco obteve decréscimo (préx=16.40, \pm 2.88 e pós $x=15.40, \pm 2.70)$.

\section{Discussão}

\section{Desenvolvimento motor e dificuldades motoras}

Os participantes apresentaram maior severidade nas habilidades de Lançar e Receber e nas habilidades de Equilíbrio estático e dinâmico. Nas habilidades de Destreza Manual foram apresentadas menores dificuldades. Este resultado diverge dos achados por Silva (2009), em que ao avaliar com o MABC em 406 escolares com idade entre 7 e 10 anos, dos quais $56.9 \%$ eram meninas e $43.1 \%$ eram meninos em Florianópolis/SC, as principais dificuldades de movimento foram nas habilidades de Destreza Manual seguidas das habilidades de Equilíbrio e por fim as habilidades com bola. Ao comparar os resultados deste estudo com outras investigações prévias, os dados sugerem uma tendência das crianças brasileiras a apresentarem uma média de desempenho das habilidades motoras abaixo do nível esperado para a idade (França, 2008; Silva, 2009).Nesta mesma perspectiva, Valentini e Rudisill (2004) avaliaram o desenvolvimento motor de escolares de 5 a 10 anos de idade com e sem atrasos no desempenho motor. Os participantes $(n=104)$ foram avaliados por meio do TGMD (Test of Gross Motor Development) antes e após a intervenção. Os resultados revelaram que as crianças com e sem atrasos motores que participaram da intervenção demonstraram mudanças significativas no desempenho das habilidades mo-

Tabela 5 - Pontuação total e por categoria do SPPC pré e pós-programa de intervenção motora.

\begin{tabular}{|c|c|c|c|c|c|c|c|c|}
\hline & \multicolumn{3}{|c|}{ PRÉ } & \multicolumn{3}{|c|}{ Pós } & \multicolumn{2}{|c|}{ Wilcoxon } \\
\hline & $x$ & $\mathrm{dp}$ & $\mathrm{m}_{\mathrm{d}}$ & $x$ & $\mathrm{dp}$ & $\mathrm{m}_{\mathrm{d}}$ & Z & $\mathbf{P}$ \\
\hline $\begin{array}{l}\text { Competência } \\
\text { Escolar }\end{array}$ & 16.67 & 4.64 & 17.00 & 15.08 & 3.42 & 14.00 & -1.720 & 0.085 \\
\hline $\begin{array}{l}\text { Aceitação } \\
\text { Social }\end{array}$ & 18.50 & 4.64 & 20.00 & 17.92 & 3.37 & 18.50 & -0.476 & 0.634 \\
\hline $\begin{array}{l}\text { Competência } \\
\text { Atlética }\end{array}$ & 16.83 & 3.81 & 18.00 & 16.92 & 2.74 & 16.00 & -0.045 & 0.964 \\
\hline $\begin{array}{l}\text { Aparência } \\
\text { Física }\end{array}$ & 19.58 & 3.20 & 19.00 & 16.42 & 5.82 & 16.50 & -1.897 & 0.058 \\
\hline Comportamento & 17.58 & 4.81 & 19.00 & 15.42 & 3.82 & 15.50 & -1.784 & 0.074 \\
\hline $\begin{array}{l}\text { Mais-Valia } \\
\text { Global }\end{array}$ & 20.33 & 3.70 & 21.00 & 17.17 & 4.71 & 17.50 & -2.304 & 0.021 \\
\hline $\begin{array}{c}\text { Autoconceito } \\
\text { Total }\end{array}$ & 109.50 & 16.30 & 110.50 & 98.92 & 17.22 & 99.50 & -2.708 & 0.007 \\
\hline
\end{tabular}


Tabela 6. Pontuação total e por categoria do SPPC pré e pós programa de intervenção motora no Grupo de TDC (GT) e Grupo de Risco para TDC (GR).

\begin{tabular}{|c|c|c|c|c|c|c|c|c|c|}
\hline & & \multicolumn{3}{|c|}{ PRÉ } & \multicolumn{3}{|c|}{ Pós } & \multicolumn{2}{|c|}{ Wilcoxon } \\
\hline & & $x$ & $d p$ & $m_{d}$ & $x$ & $d p$ & $m_{d}$ & z & $\mathbf{P}$ \\
\hline \multirow{2}{*}{$\begin{array}{l}\text { Competência } \\
\text { Escolar }\end{array}$} & $\mathrm{GT}$ & 18.28 & 4.75 & 19.00 & 16.14 & 3.53 & 17.00 & -1.549 & 0.121 \\
\hline & GR & 14.40 & 3.78 & 14.00 & 13.60 & 2.97 & 14.00 & -0.736 & 0.461 \\
\hline \multirow{2}{*}{ Aceitação Social } & $\mathrm{GT}$ & 17.57 & 6.00 & 20.00 & 17.86 & 3.48 & 20.00 & -0.271 & 0.786 \\
\hline & GR & 19.80 & 0.84 & 20.00 & 18.00 & 3.60 & 18.00 & -0.730 & 0.465 \\
\hline \multirow{2}{*}{$\begin{array}{c}\text { Competência } \\
\text { Atlética }\end{array}$} & GT & 17.14 & 4.56 & 18.00 & 18.00 & 2.38 & 19.00 & -0.527 & 0.598 \\
\hline & GR & 16.40 & 2.88 & 18.00 & 15.40 & 2.70 & 15.00 & -0.674 & 0.500 \\
\hline \multirow{2}{*}{ Aparência Física } & GT & 19.43 & 2.64 & 19.00 & 18.00 & 5.66 & 16.00 & -0.542 & 0.588 \\
\hline & GR & 19.80 & 4.21 & 21.00 & 14.20 & 5.89 & 12.00 & -1.826 & 0.068 \\
\hline \multirow{2}{*}{ Comportamento } & $\mathrm{GT}$ & 19.14 & 5.18 & 20.00 & 15.57 & 4.89 & 16.00 & -2.207 & $0.027^{*}$ \\
\hline & GR & 15.40 & 3.65 & 15.00 & 15.20 & 2.05 & 15.00 & -0.135 & 0.892 \\
\hline \multirow{2}{*}{ Mais-Valia Global } & $\mathrm{GT}$ & 20.00 & 4.08 & 21.00 & 18.14 & 5.11 & 18.00 & -1.476 & 0.140 \\
\hline & GR & 20.80 & 3.42 & 21.00 & 15.80 & 4.21 & 15.00 & -1.841 & 0.066 \\
\hline \multirow{2}{*}{ TOTAL } & $\mathrm{GT}$ & 111.57 & 20.35 & 111.0 & 103.71 & 19.00 & 108.00 & -1.612 & 0.107 \\
\hline & GR & 16.60 & 9.55 & 110.0 & 92.20 & 13.33 & 93.00 & -2.023 & $0.043^{*}$ \\
\hline
\end{tabular}

toras, enquanto que as crianças do grupo sem intervenção não evidenciaram melhorias neste período.

Em estudo anterior, Valentini (2002b) objetivou determinar a influência de uma intervenção motora no desenvolvimento motor de crianças de 6 a 10 de idade que sinalizaram atrasos motores previamente identificados, com 91 crianças em dois grupos distintos (intervenção, $n=14$; e controle $n=50$ ); os resultados demonstraram que a intervenção promoveu modificações significativas e positivas, oportunizando vivências de atividades motoras desafiadoras para as crianças. Estes dados fortalecem o entendimento de que as habilidades motoras não emergem naturalmente e de que é necessário propiciar às crianças instrução adequada, métodos sistemáticos e consistentes de ensino, pois o ensino é fundamental para o desenvolvimento motor, tendo-se em vista as dificuldades neste contínuo processo estarem comumente relacionadas à falta de oportunidade de prática, à falta de instrução adequada ou ainda à falta de experiência motora.

Os estudos que analisam o desempenho de crianças com TDC em diversas tarefas têm demonstrado maiores dificuldades na execução das atividades por esta população, a qual apresenta maior variabilidade e inconsistência de resposta (Oliveira, 2003; Schoemaker \& cols., 2006). Astill e Utley (2006) demonstraram que na tarefa de segurar uma bola de tênis com as duas mãos, as crianças com TDC de
7 e 8 anos apresentaram maior congelamento dos graus de liberdade nos membros superiores, demonstrando rigidez na performance do movimento de agarrar. Além do mais, a maior variabilidade intra-indivíduos relacionou-se à falta de flexibilidade em realizar ajustes corporais no acoplamento dos membros.

Toda tarefa nova em cuja execução não tenha havido prática prévia ou possibilidade de transferência de aprendizagem anterior será relativamente complexa para o aprendiz, principalmente para as crianças com TDC, considerando suas maiores dificuldades perceptivas e de coordenação motora (Nunomura \& Nista-Piccolo, 2005). Em concordância com estes resultados, também se ressalta o estudo realizado com a bateria MABC em crianças brasileiras de 9 e 10 anos de escola pública e particular de Teixeira, Gimenez, Oliveira e Dantas (2010), confirmando maiores dificuldades nas habilidades manipulativas onde os valores de média do escore parcial de destreza manual para as crianças de escola pública foi de 9.35 e de escola particular 8.6.

\section{Auto conceito}

Os escolares com Transtorno de Desenvolvimento da Coordenação (TDC) participantes deste estudo apresentaram um autoconceito geral mais positivo que aos participantes com risco para TDC. Este achado não reflete 
as características próprias do perfil de crianças com dificuldades motoras apresentadas na literatura (Maturano, 1997; Hay, Aschaman, \& Kraayenoord, 1998; Martini \& Burochovith, 1998). No estudo de Cia e Barham (2008), cujo objetivo era estabelecer relação entre autoconceito e desempenho atlético de crianças escolares, 58 crianças de $5^{a}$ e $6^{a}$ séries do ensino fundamental foram avaliadas usando o Self-description Questionnaire1 (SDQ1), concluindo que o autoconceito estava positivamente correlacionado com o desempenho atlético dos escolares. Tais resultados vão ao encontro com achados de outras pesquisas, que apontam a influência mútua entre o desenvolvimento motor e o desenvolvimento do autoconceito de crianças em fase escolar (Maturano, 2004). Nesses estudos os resultados sugerem a presença de impacto negativo das dificuldades motoras no autoconceito das crianças. Possivelmente, esta correlação existe porque as crianças com melhor autoconceito são mais confiantes, são mais persistentes em suas atividades atléticas, não têm medo de errar; por isso não temem competições, se relacionam melhor com outras pessoas (colegas, pais e professores) e se responsabilizam pelos seus fracassos e sucessos (Bacete \& Betoret, 2000).Além disso, o autoconceito mais positivo também pode estar associado a uma maior autonomia e competência, variáveis estas consideradas por Deci, Hodges, Pierson e Tomassone (1992) como fatores motivacionais importantes para o aprendizado de novas habilidades.

\section{Influência do programa de intervenção motora}

O presente programa de intervenção motora demonstrou influenciar positivamente o desenvolvimento motor dos escolares com risco e indicativo de TDC, corroborando com a maioria dos estudos relevantes sobre intervenção motora em crianças com TDC ou em mesmas condições (Pless \& Carlsson, 2000; Ferreira \& cols., 2006; Santos, Dantas \& Oliveira, 2004; Souza, Ferreira, Catuzzo, \& Corrêa, 2007). Estudos internacionais apontam uma maior eficácia quandoo programa interventivo ocorre com frequência de três a cinco vezes por semana, realizado em grupos homogêneos ou em ambiente familiar (Pless \& Carlsson, 2000; Ferreira, Nascimento, Apolinário, \& Freudenhein, 2006). Santos, Dantas e Oliveira (2004) destacam que para que a criança atinja essas transformações com naturalidade é fundamental que vivencie as mais variadas formas de experiência motoras, ressaltando a importância do movimento para que a criança consiga com êxito o amplo domínio do seu corpo nas mais diversas habilidades motoras fundamentais, que só irá ocorrer por meio de vivências motoras, lúdicas e desafiadoras, nos principais ambientes de convívio da criança. Dificuldades no desempenho de habilidades motoras fundamentais diminuem as chances de participação da criança em atividades motoras escolares, diminuindo seu engajamento motor e a participação futura em atividades que asseguram a qualidade de vida (Valentini \& Rudisill, 2004) e com intervenção, as crianças podem adquirir certas habilidades, mas as atividades motoras novas continuam a ser um problema (Missiuna, 2003).

Durante as atividades, a criança com dificuldade motora enfrenta a rejeição dos companheiros, os quais lhe negam participação nas atividades esportivas, no que resulta em frustração e aceitação. Vivências nas tarefas motoras, o suporte do professor e o próprio aumento da competência motora podem levar as crianças a demonstrarem mudanças positivas na forma como se percebem (Harter, 1982; Valentine, 2002). Práticas adequadas, com persistência temporal na atividade e um significado para a criança são fundamentais para o desenvolvimento desta (França, 2008). Nesta mesma direção, Harter (1978) salienta que quanto mais experiências são vivenciadas pela criança em uma atividade, maiores oportunidades ela terá para desenvolver o senso de autoconceito. Portanto, a participação das crianças no Programa Interventivo, de forma sistemática durante 21 aulas, intensificou as vivências nas habilidades motoras repercutindo positivamente nas percepções de competência motora das crianças. Porém, um programa isolado de atividade física não garante o desenvolvimento da criança, bem como não consegue atingir a família a mudar culturalmente seus hábitos de vida (Damaso, 2001). Portanto, analisando como essas crianças chegaram a termos de avaliação motora e como elas se encontraram no final do Programa Interventivo, os resultados sinalizam que este teve um impacto positivo sobre elas, repercutindo em maior senso de competência motora.

É possível concluir que o desenvolvimento motor dos participantes denotou "dificuldades motoras significantes", e essas dificuldades foram mais severamente detectadas nas habilidades de lançar e receber, e nas tarefas de equilíbrio estático. Por meio das análises dos resultados pode-se perceber que o programa de intervenção motora implementado demonstrou influenciar positivamente no desenvolvimento motor tanto para das crianças com risco como com indicativo para TDC. Portanto, esses resultados evidenciaram que as atividades motoras desenvolvidas no Programa Interventivo foram desenvolvimentistas; oportunizaram uma vivência motora enriquecedora, baseada em proposta metodológica adequada e criando perspectivas futuras na participação efetiva das crianças em práticas esportivas sistemáticas, aumentando assim o repertório motor destas.

Enfatiza-se ainda, que quando se trabalha com crianças com TDC essas condições são muito mais importantes. De modo geral, notou-se que o TDC constitui um complexo transtorno motor ainda pouco conhecido na comunidade escolar e que leva a criança a graus variáveis de comprometimento na vida social, emocional, escolar e familiar, resultando em dificuldades globais em seu desenvolvimento. Portanto, o conhecimento do impacto negativo desse transtorno na qualidade de vida dessas crianças e de seus familiares impõem a necessidade de intervenções efetivas nessa população.

Nesse sentido, ao final do estudo, o autoconceito apresentado pelos participantes previamente ao programa interventivo foi contrário ao descrito na literatura, onde se 
espera que o baixo desempenho motor leve a um baixo autoconceito. Pode-se especular que o alto senso de adequação apresentado pelos participantes do estudo deve-se ao fato de que no seu cotidiano escolar as crianças não experimentem situações de avaliação de seu desempenho e que as aulas de Educação Física tenham um caráter demasiado recreativo e lúdico. Nesta perspectiva, a diminuição da pontuação do autoconceito seria uma maior adequação do senso de percepção dos participantes.

Como fatores limitantes desta pesquisa é possível apontar algumas variáveis que, de alguma forma, podem influenciar os resultados obtidos e não foram controladas. Entre essas variáveis destacam-se o processo maturacional, os hábitos associados ao estilo de vida, bem como a incapacidade dos pesquisadores de fornecer o mesmo nível de motivação aos participantes do estudo. As atividades cotidianas da criança, como estímulos motores, moradia, tempo em que passa assistindo à televisão ou no computador, bem como atividades extracurriculares que a criança pratica também devem ser observadas, pois o tipo de atividades e experiências que a criança acumula podem ser importantes tanto na vida cotidiana como para o aprendizado de novas habilidades. Sugere-se o controle dessas variáveis para novas pesquisas, bem como ouso do grupo controle.

\section{Referências}

American Psychiatric Association. [APA] (1995). DSM-IV. Manual diagnóstico e estatístico de transtornos mentais. (D. Batista, Trad., $4^{\mathrm{a}}$ ed.). Porto Alegre: Artes Médicas.

Astill, S. \& Utley, A. Two-Handed Catching in Children with Developmental Coordination Disorder. Motor Control, 10, p.109124, 2006.

Bacete, F. J.G. \& Betoret, F.D. (2000). Motivación, aprendizaje y rendimiente escolar. Revista Española de Motivación y Emoción, 1, 55-65.

Bandeira, D., Reppold, C., Plüger, D., \& Facio, A. (2003). Escala Harter de Auto-Estima: adaptação para o contexto brasileiro. Anais do I Congresso Brasileiro de Avaliação Psicológica e IX Conferência Internacional de Avaliação Psicológica. Campinas: IBAP.

Byrne, B.M. (1984). The general/academic self-concept nomological network: A review of construct validation research. Review of Educational Research, 54, 427-456.

Campos, A.A. (2004). Adaptação Cultural da Escala "Perfil de Autopercepção para Crianças". Dissertação de mestrado, Instituto de Psicologia da UFRJ, Rio de Janeiro, RJ.

Cantell, M.H., Smyth, M.M., \& Ahonen, T.P. (1994). Clusminess in adolescence: educational, motor and social outcomes of motor delay detected at five years. Adapted Physical Activity Quartely,11, 115-129.
Crawford, S.G., Wilson, B.N., \& Dewey, D. (2001). Identifying developmental coordination disorder: Consistency between tests. Physical and Occupational Therapy in Pediatrics, 20, 29-50.

Croce, R. V., Horvat, M., \& Mccarthy, E. (2001). Reliability and concurrent validity of the movement assessment battery for children. Perceptual and Motor Skills, 93(1), 275-80.

Damaso, A. (2001). Nutrição e exercícios na prevenção de doenças (1 ${ }^{\text {a }}$.ed.). Rio de Janeiro: MEDSI.

Deci, E. L., Hodges, R., Pierson, L., \& Tomassone, J. (1992). Autonomy and competence as motivational factors in students with learning disabilities and emotional handicaps. Journal of Learning Disabilities, 25, 457-471.

Dewey, D., Kaplan, J.B., Crawford, S.G., \& Wilson, B.N. (2002). Development coordination disorder: associated problems in attention, learning, and psychosocial adjustment. Human movement science, 21, 905-918.

Dewey, D., Wilson, B. N. (2001). Developmental Coordination Disorder: What is it. Physical \& OccupationTherapyin Pediatrics, 20(3), 5-27.

Ferreira, L.F., Nascimento, R.O., Apolinário, M.R., \& Freudenhein, A.M. (2006). Desordem da Coordenação do Desenvolvimento. Motriz,12(3), 283-292.

França, C. (2008). Desordem Coordenativa Desenvolvimental em Crianças de 7 e 8 anos de idade. Dissertação de mestrado, CEFID, Universidade do Estado de Santa Catarina, Florianópolis, SC.

Gallahue, D.L., \& Ozmun, J.C. (2005). Compreendendo o desenvolvimento motor: bebês, crianças, adolescentes e adultos (3a. ed.). São Paulo: Phorte.

Geuze, R.H., Jongmans, M.J., Scoemaker, M., \& Smits-Engelsman, B.C. (2001). Clinical and research diagnostic criteria for developmental coordination disorder: a review and discussion. Human Movement Science, 20, 7-47.

Gillberg, A.M. (2000). Children with preschool minor neurodevelopmental disorders. IV: Behavior and school achievemenat age 13. Developmental Medicine and Child Neurology, 31, 14-24.

Hands, B. (2008). Changes in Motor Skill and Fitness Measures Among Children With High and Low Motor Competence: A FiveYear Longitudinal Study. Sports Medicine Australia.

Harter, S. (1978). Effectunce motivation reconsided: toward a developmental model. Human Development, 1, 34-64.

Harter, S. (1982). The Perceived Competence Scale for Children. Child Development, 53 (1), 87-97. 
Harter, S. (1984). The Pictorial Scale of Self Perception and Social Acceptance for Young Children. University of Denver.

Harter, S. (1985). Manual for the self-perception profile for children. University of Denver.

Hay, I., Ashman, A.F., \& Kraayenoord, C. (1998).Educational characteristics of students with high or low self-concept. Psychology in the school, 4 (35), 391-400.

Hay, J., Hawes, R., \& Faught, B. E. (2004).Evaluation of a screening instrument for Developmental Coordination Disorder, Journal of Adolescent Health, 34, 308-313.

Hellgren, L., Gillberg, I.C., Bagenholm, A., \& Gillberg, C. (1994). Children with deficits in attention, motor control and perception (DAMP) almost grown up: psychiatric and personality disorders at age 16 years. J. Child. Psychol. Psychiatry, v. 3, 1255-1271.

Henderson. S.E. \& Sugden. D.A. (1992). Movement Assessment Battery for Children-MABC. London: Psychological Corporation.

Henderson, S.E., Sugden, D.A., \& Barnett, A. (2007). Movement Assessment Battery for Children 2 - MABC2. London.

Krug, D.F. (2009). Metodologia do Ensino em Educação Física - O Spectrum de Muska Mosston e uma Nova Visão!. Livraria Jurídica, Lisboa,

Martini, M.L. \& Boruchovitch, E. (1998). As atribuições de causalidade, o desenvolvimento infantil e o contexto escolar. PSICO-USF, 4(2), 23-36.

Maturano, E. M. (1997). A criança, o insucesso escolar precoce e a família: condições de resiliência e vulnerabilidade. Em E.M. Maturano, S.R. Loureiro, \& A. W. Zuardi (Orgs.), Estudos em Saúde Mental (pp. 132-145). Ribeirão Preto: Comissão de PósGraduação em Saúde Mental - FMRP/USP.

Maturano, E.M. (2004). Fatores de risco e proteção no desenvolvimento sócio-emocional de crianças com dificuldades de aprendizagem. Em E.G. Mendes, M.A. Almeida \& L.C.A. Williams (Orgs), Avanços recentes em Educação Especial (pp. 159-165). São Carlos: EDUFSCar.

Missiuna, C. (2003). Crianças com Transtorno do Desenvolvimento da Coordenação: em casa e na sala de aula (Magalhães, L. C., Trad.). Ontario, Canada: Can Child, Center for Childhood Disability Research

Nunomura, M. \& Nista-Piccolo, V.L. (2005). Compreendendo a ginástica artística. Phorte, 17-52.

Novaes, J.J. (2003). Tradução para o português e validação das escalas: Locus de Controle para Crianças (NSLC) e Perfil de Autopercepção para Crianças (SPPC). Dissertação de mestrado,
Faculdade de Ciências Médicas, Universidade Estadual de Campinas.

Oliveira, M.A., Loss, J.F., \& Petersen, R.D.S. (2005). Controle de força e torque isométrico em crianças com DCD. Revista Brasileira de Educação Física Esp,19 (2), 89-103.

Oliveira, M.A. (2003). Controle de força e torque isométrico em crianças com e sem desordem coordenativa desenvolvimental. Tese de doutorado, Escola Superior de Educação Física, Universidade Federal do Rio Grande do Sul, Porto Alegre, RS

Pless, M. \& Carlsson, M. (2000) Effects of Motor Skill Intervention on Developmental Coordination Disorder: a Meta-Analysis. Adapted Physical Activity Quarterly,17, 381-401.

Polatajko, H.A. (2003). Clinical trial of the process-oriented treatment approach for children with developmental co-ordination disorder. Developmental Medicine and Children Neurology, 37, 310-319.

Santos, S., Dantas, L., \& Oliveira, J. A. (2004). Desenvolvimento motor de crianças, de idosos e de pessoas com transtornos da coordenação. Revista Paulista de Educação Física, 18, 33-44.

Schoemaker, M.M., Kloetheleen, A., Reinders-Messelink, H., Verheij, N., Wilson, B. N., Flapper, B., \& Arendde Kloet, W. (2006). Evaluation of the Developmental Coordination Disorder Questionnaire (DCDQ) as a screening instrument. Developmental Medicine \& Child Neurology, 48, 668-673.

Segal, R., Mandich, A., Polatajko, H., \& Cook, J.V. (2002). Stigma and its management: a pilot study of parental percetions of the experiences of children with Developmental Coordination Disorder: The American Journal of Occupational Therapy, 56, 422-428.

Silva, J. (2009). Desempenho motor, desempenho acadêmico e senso de auto-eficácia de escolares do ensino fundamental. Dissertação de mestrado, CEFID, Universidade do estado de Santa Catarina, Florianópolis, SC.

Sisto, F., Bartholomeu, D., Rueda, F., \& Fernandes, D. (2004). Autoconceito e emoções. Psiquilíbrios, 10,68-74.

Souza, C.D., Ferreira, L., Catuzzo, M.T., \& Corrêa, U.C. (2007). O teste $A B C$ do movimento em crianças de ambientes diferentes. Revista Portuguesa de Ciência e Desporto, 7 (1), 36-47.

Teixeira, P.C. (2008). Tecido Circense: A influência de um programa de intervenção motora no desenvolvimento motor, percepção de competência e ansiedade de crianças. Dissertação de mestrado em Educação Física, Centro de Ciências da Saúde, Universidade Estadual de Maringá, Maringá, PR.

Teixeira, R., Gimenez, R., Oliveira, D.L., \& Dantas, L.E.P.B.T. (2010). Dificuldades Motoras na Infância: Prevalência e Relações com as Condições Sociais e Econômicas. Science in Health,1, 24-33. 
Thomas, J. R. \& Nelson, J.K. (2002). Métodos de pesquisa em atividade física (3a.ed.). Porto Alegre: Artmed.

Valentini, N.C. (2002a). A influência de uma intervenção motora no desempenho motor e na percepção de competência de crianças com atrasos motores. Revista Paulista de Educação Física, 16 (1), 61-75.

Valentini, N.C. (2002b). A influência de uma Intervenção motora com contexto de motivação para a maestria no desenvolvimento motor de crianças portadoras e não de necessidades especiais: uma perspectiva inclusiva. Em Anais do III Seminário de Comportamento Motor. RS: Gramado.

Valentini, N.C. (2002c). Percepções de competência e desenvolvimento motor de meninos e meninas: um estudo transversal. Revista Movimento, 1, 9-20.

Valentini, N.C., Coutinho, M.T.C., Pansera, S.M., Santos, V.A.P., Vieira, J.L.L., Ramalho, M.H., \& Oliveira, M.A. (2012). Prevalência de déficits motores e desordem coordenativa desenvolvimental em crianças da região Sul do Brasil. Revista Paulista de Pediatria, 30 (3), 377-84.
Valentini, N.C. \& Rudisill, M.E. (2004). Motivational climate, motorskill development and perceived competence: two studies of developmental delayed kindergarten children. Journal of Teaching in Physical Education, 23, 216-234.

Vallerand, R.J. (1989). Versus e methodologie de validation transculturelle de questionnaires psychologiques: implications pour La recherché em language française. Canadian Psychologie, 30 (4), 662-680.

Villwock, G. (2005). Percepção de competência e participação de crianças na atividade física. Revista Perfil, 2.

Wright, H. C. \& Sugden, D.S. (1996).The nature of developmental coordination disorder: inter and intra-group differences. Adapted Physical Activity Quarterty, 13, 356-371.

\section{Sobre os autores}

Thais Silva Beltrame (tsbeltrame@gmail.com)

Profa. Dra . do Programa de Pós-Graduação em Ciências do Movimento Humano da Universidade do Estado de Santa Catarina.

Fernando Luiz Cardoso (fernando.cardoso@udesc.br)

Prof. Dr. do Programa de Pós-Graduação em Ciências do Movimento Humano e do Programa de Pós-Graduação em Educação da Universidade do Estado de Santa Catarina.

Juliano Maestri Alexandre (julianom.alexandre@gmail.com)

Mestrando do Programa de Pós-Graduação em Ciências do Movimento Humano da Universidade do Estado de Santa Catarina

Carla Simon Bernardi (carlinhasimon@hotmail.com)

Mestre em Ciências do Movimento Humano da Universidade do Estado de Santa Catarina. 
